\title{
Review Article \\ Pathogenic Roles of Macrophage Migration Inhibitory Factor during Dengue Virus Infection
}

\author{
Yung-Chun Chuang, ${ }^{1,2}$ Hong-Ru Chen, ${ }^{2,3}$ and Trai-Ming Yeh ${ }^{1,2,3}$ \\ ${ }^{1}$ Department of Medical Laboratory Science and Biotechnology, Medical College, National Cheng Kung University, \\ Tainan 70101, Taiwan \\ ${ }^{2}$ Center of Infectious Disease and Signaling Research, Medical College, National Cheng Kung University, Tainan 70101, Taiwan \\ ${ }^{3}$ The Institute of Basic Medical Sciences, Medical College, National Cheng Kung University, Tainan 70101, Taiwan
}

Correspondence should be addressed to Trai-Ming Yeh; today@mail.ncku.edu.tw

Received 24 October 2014; Revised 14 January 2015; Accepted 5 February 2015

Academic Editor: Robert Anderson

Copyright (C) 2015 Yung-Chun Chuang et al. This is an open access article distributed under the Creative Commons Attribution License, which permits unrestricted use, distribution, and reproduction in any medium, provided the original work is properly cited.

\begin{abstract}
Dengue virus (DENV) infection is the most common cause of viral hemorrhagic fever, which can lead to life-threatening dengue hemorrhagic fever/dengue shock syndrome (DHF/DSS). Hemorrhage and plasma leakage are two major hallmarks of DHF/DSS. Because the mechanisms causing these pathogenic changes are unclear, there is no effective therapy against DHF/DSS. In this review, we focus on the possible pathogenic effects of a pleiotropic cytokine, macrophage migration inhibitory factor (MIF), on the pathogenesis of DENV infection. MIF is a critical mediator of the host immune response and inflammation, and there is a correlation between the serum levels of MIF and disease severity in dengue patients. Furthermore, MIF knock-out mice exhibit less severe clinical disease and lethality. However, the role of MIF in the pathogenesis of DHF/DSS is not limited to immune cell recruitment. Recent evidence indicates that DENV infection induced MIF production and may contribute to vascular hyperpermeability and viral replication during DENV infection. The expression of both adhesion and coagulation molecules on MIF-stimulated monocytes and endothelial cells is also increased, which may contribute to inflammatory and anticoagulatory states during DHF/DSS. Therefore, blocking MIF production or its function may provide a solution for the treatment and prevention of DHF/DSS
\end{abstract}

\section{Introduction}

1.1. The Structure and Expression of Macrophage Migration Inhibitory Factor (MIF). Macrophage migration inhibitory factor (MIF), which is also known as glycosylation-inhibiting factor (GIF), L-dopachrome isomerase, or phenylpyruvate tautomerase, was first identified as a cytokine inhibiting the random migration of macrophages $[1,2]$. MIF is an evolutionarily highly conserved protein that is abundantly expressed in human and other species. MIF is composed of 114 amino acids, producing a cytokine of $12.5 \mathrm{kDa}$ [3]. In contrast to other cytokines, MIF possesses a unique catalytic function as a tautomerase. Under physiologic conditions, MIF exists as a trimer consisting of three identical subunits, an arrangement that confers a three-dimensional structure of MIF resulting in a catalytic site located in the intermonomeric pocket [4]. Although T cells were first identified as the main source of this cytokine, MIF is now known to be widely expressed in various cell types, including monocytes, macrophages, hepatocytes, and endothelial cells $[1,2,5-8]$. The secretion of MIF by macrophages is induced by low levels of glucocorticoids and is suggested to counteract the inhibitory effects of glucocorticoids in the regulation of the immune system [9-11]. Recently, it has been revealed that activated platelets are also a source of MIF [12].

1.2. The Activating Mechanism of MIF. Despite its wide tissue distribution, the secretion of MIF is tightly regulated by relevant triggers, such as inflammation and hypoxia. It has long been known that the secretion of MIF is correlated to infectious diseases, autoimmune diseases, heart and vascular diseases, and cancer. After secretion, MIF activates downstream pathways in an autocrine or paracrine manner. 
The first identified receptor of MIF was CD74, the membraneexpressed form of invariant chain and an MHC class II chaperone [13]. Due to the lack of an intracellular domain, the activation of CD74 by MIF relies on the recruitment of coreceptors such as CD44 or CXCR2 and CXCR4 [14]. In a recent study, another chemokine receptor, CXCR7, has been shown to engage with MIF to modulate tumor metastasis [15]. CD44 is required for transmitting the MIF/CD74 signal by relaying the Src tyrosine kinase-mediated phosphorylation of serine on the cytosolic tail of CD74 and CD44; this phosphorylation then activates the downstream ERK/MAPK and PI3K/Akt pathways [16-18]. In addition to CD74, the direct binding of MIF and CXCR2 or CXCR4 was also observed to induce calcium influx and the rapid activation of integrins by Gi-coupling [19]. CXCR7 could be activated by MIF to initiate the Akt pathway to regulate platelet apoptosis [20]. In addition to transmitting signals through receptors, MIF can be endocytosed into the cytosol and interact with JAB-1 to inhibit the activity of AP-1 proteins [21]. Secreted MIF is capable of activating $\mathrm{T}$ cells and macrophages to produce proinflammatory cytokines, including tumor necrosis factor- (TNF-) $\alpha$, interleukin- (IL-) $1 \beta$, IL-2, IL-6, IL-8, and interferon- (IFN-) $\gamma$, leading to inflammatory responses. Therefore, MIF plays a pivotal role in the modulation of both innate and adaptive immune responses $[8,22]$.

1.3. The Role of MIF in Inflammatory Diseases. The first evidence implicating the pathogenic roles of MIF in inflammatory diseases dates back to two decades [23]. Employing a mouse endotoxic shock model, recombinant MIF was found to greatly enhance lethality when coinjected with lipopolysaccharide (endotoxin), whereas an anti-MIF antibody conferred full protection against lethal endotoxemia. Elevated levels of MIF were subsequently found in the plasma of patients with sepsis and septic shock and were positively correlated with the sepsis severity $[24,25]$. Blockage of MIF with neutralizing antibodies or the tautomerase inhibitor 4,5dihydro-3-(4-hydroxyphenyl)-5-isoxazole acetic acid, methyl ester (ISO-1) increases the survival rate in septic mice models $[26,27]$. In addition to sepsis, MIF knock-out $\left(\mathrm{Mif}^{-/-}\right)$mice show reduced mortality compared to wild-type mice after Toxoplasma gondii infection, indicating that MIF is also involved in the pathogenesis of infection by this protozoan [28]. In addition to the pathogenic roles of MIF in acute infection, MIF is also essential for the pathogenesis of chronic diseases, such as autoimmune and cardiovascular diseases, as well as cancer [29-33]. However, unlike the case in cancer and autoimmune diseases, MIF may have a protective effect in the heart during ischemia or other cardiovascular diseases $[31,34]$.

1.4. MIF in Viral Infection. In addition to bacterial infection, elevated MIF levels are also observed in viral infections, such as those caused by influenza virus, human immunodeficiency virus (HIV), Ebola virus, and dengue virus (DENV) [3539]. DENV infection generally causes mild symptoms such as fever, headache, and muscle and joint pain, comprising dengue fever (DF). In some cases, especially during secondary infection with a different serotype of DENV, DENV infection may progress to dengue hemorrhagic fever (DHF) or dengue shock syndrome (DSS) [40]. DHF is a severe febrile disease characterized by abnormalities in homeostasis and increased capillary leakage that can progress to hypovolemic shock (DSS). Although the mechanisms causing hemorrhage and vascular leakage in DHF/DSS remain unclear, there are few hypotheses for severe dengue disease, the most well known of which is antibody-dependent enhancement (ADE) [41]. According to ADE, the antibodies generated in the first DENV infection have no protective effect but rather enhance the second infection of a different DENV serotype through the $\mathrm{F} c \gamma$ receptor of such host cells as macrophages. Other hypotheses, including cytokine production and complement activation, and autoantibody immunopathogenesis also play crucial roles that might contribute to severe DHF/DSS during DENV infections [42-44]. Nevertheless, it is known that the serum levels of MIF in dengue patients correlate with disease severity and clinical outcome [39]. Furthermore, $\mathrm{Mif}^{-/-}$mice exhibit less severe clinical disease, lower viremia, and lower viral load in the spleen than wild-type mice after DENV infections [38]. Blocking MIF also rescues DENV-induced vascular leakage, indicating the importance of MIF in the pathogenesis of DENV infection [45]. Therefore, the current review focuses on the possible pathogenic roles of MIF during DENV infection.

\section{MIF Production Induced by DENV Infection}

DENV infection, but not stimulation with UV-inactivated DENV, induces MIF secretion in a dose- and time-dependent manner in the human epithelial cell line A549 [46]. A MIF promoter assay and reverse transcription polymerase chain reaction (RT-PCR) demonstrate that MIF gene transcription is activated during DENV infection. Furthermore, DENV infection induces NF- $\kappa$ B activation, and the DENV-induced production of MIF is inhibited in the presence of the NF- $\kappa \mathrm{B}$ inhibitor dexamethasone or curcumin. In addition, different cell types, such as primary human vascular endothelial cells (HUVECs) and peripheral blood mononuclear cells (PBMCs), have different abilities to release MIF after DENV infection. Interestingly, DENV infection of the human monocytic cell line THP-1 and PBMCs and the subsequent MIF production are enhanced in the presence of antibodies against DENV. This phenomenon may due to an ADE effect. Based on $\mathrm{ADE}$, antibodies against DENV may enhance virus uptake by macrophages through the Fc receptor, thus enhancing viral replication and altering the cytokine production profile [47]. Therefore, it is possible that DENV infection of human cells induces NF- $\kappa \mathrm{B}$ activation, which leads to MIF production and that this process is enhanced in the presence of preexisting anti-DENV antibodies.

\section{MIF in DENV Replication}

It is known that MIF is involved in the replication of many different viruses during infection. For example, in HIV-1 infection, MIF neutralization can diminish HIV-1 replication in human PBMCs [48] (Table 1). In $\mathrm{Mif}^{-/-}$mice, West 
TABLE 1: Effects of MIF in the replication of different viruses.

\begin{tabular}{lcccc}
\hline Virus & Model & Treatment & Viral replication & Reference \\
\hline HIV-1 & HIV-1-infected PBMCs & $\begin{array}{c}\text { Anti-MIF antibodies } \\
\text { rMIF }\end{array}$ & $\begin{array}{c}\text { p24 expression } \downarrow \\
\text { p24 expression } \uparrow\end{array}$ \\
\hline WNV & WNV-infected $\mathrm{Mif}^{-/-}$mice & [48] & WNV E-mRNA $\downarrow$ in brain tissues \\
\hline RRV & RRV-infected $\mathrm{Mif}^{-/-}$mice & [50] \\
\hline DENV & DENV-2-infected $\mathrm{Mif}^{-/-}$mice & & Virus PFU $\downarrow$ in ankle tissues \\
\hline
\end{tabular}

Nile virus (WNV) and Ross River virus (RRV) have shown reduced viral replication in the brain, ankle, serum, and spleen $[49,50]$. However, there is only one related reference to date indicating the importance of MIF in DENV replication [38]. In addition to lower levels of TNF- $\alpha$, IL-6, and $\mathrm{PEG}_{2}$, the viral load of DENV in $\mathrm{Mif}^{-/-}$mice is lower than that in wildtype mice. Moreover, the mortality of DENV-infected mice is delayed and the thrombocytopenia is reduced in $\mathrm{Mif}^{-/-}$mice. However, the precise mechanism by which MIF regulates DENV replication is still unknown.

Several studies have indicated that autophagy is required for optimal DENV replication [51-53] and different cytokines have different effects on autophagy formation. T helper type 1 (Th1) cytokines such as IFN- $\gamma$, IL-12, and TNF- $\alpha$ induce or promote autophagy in macrophages as well as nonimmune cells $[54,55]$. In contrast, Th2 cytokines such as IL-4, IL-10, and IL-13 appear to be antagonists of autophagy induction [56]. Because MIF can induce Th1 cytokine expression, it may contribute to autophagy formation. Recently, we found that MIF could enhance autophagy via reactive oxygen species (ROS) generation [57]. Thus, autophagy may be one of the possible pathways by which MIF may regulate DENV replication. However, whether MIF-induced autophagy plays a pivotal role in DENV replication remains to be proven.

\section{MIF in DENV-Induced Vascular Hyperpermeability}

Although elevated MIF levels were observed in diseases that feature vascular leakage, the regulation of vascular permeability by MIF was first indicated in a study of WNV infection [50]. This study suggested that brain infection with WNV might be promoted by MIF-induced increased permeability of the blood-brain barrier. MIF-induced vascular leakage was later suggested in DENV infection [45]. Recombinant MIF as well as a DENV infection-induced conditioned medium increases vascular permeability by inducing disarray of the tight junction protein $\mathrm{ZO}-1$, effects that are blocked by a MIF inhibitor (ISO-1) or CD74 knockdown. However, the mechanism by which MIF regulates the alignment of junction proteins remains unclear. Cytokines increase vascular permeability by regulating the expression and localization of junction proteins by various mechanisms. For example, VEGF induces endothelial hyperpermeability through the endocytosis of junction proteins, TNF- $\alpha$ alters vascular permeability by decreasing the mRNA content related to junction proteins, and IL- $1 \beta$ and thrombin induce the translocation of junction proteins and increase vascular permeability by regulating the arrangement of the cytoskeleton [58-62]. MIF was previously demonstrated to induce autophagy in hepatocytes [57]. Although autophagy has been suggested to regulate the turnover of junction proteins, whether it mediates vascular permeability has not yet been studied. Our recent work suggested that MIF might regulate vascular permeability through autophagy [63]. Further investigation of how MIF and autophagy regulate vascular permeability will open up promising novel therapeutic avenues to prevent DENV-induced vascular leakage.

\section{MIF in DENV-Induced Adhesion Molecule and Coagulation Molecule Expression}

It is known that MIF can induce the production of intercellular adhesion molecule-1 (ICAM-1) [64] and the coagulation molecule thrombomodulin (TM) [65]. ICAM-1 is a ligand for lymphocyte function-associated molecule-1 (LAF-1), which is expressed on the leukocyte cell surface. Through ICAM1 and LFA-1 interaction, leukocytes can bind to endothelial cells and then transmigrate into tissues. In addition, the production of coagulation and anticoagulation factors is affected by MIF in endothelial cells, THP-1 cells and Hep G2 cells [66]. Reverse transcription-polymerase chain reaction (RT-PCR), immunofluorescent staining, and flow cytometry demonstrate that recombinant MIF induces TM protein expression in endothelial cells and THP-1 cells, and this effect is blocked by a MIF-neutralizing antibody [66]. TM is a transmembrane glycoprotein that is expressed on the surface of vascular endothelial cells as well as monocytes and a variety of other cell types. TM can compete with fibrinogen to bind to thrombin and inhibit fibrin formation, thereby interfering with coagulation. The thrombin-TM complex can also activate protein $\mathrm{C}$ (APC), which can digest the active clotting factors $\mathrm{Va}$ and VIIIa to inhibit further thrombin formation. Thus, TM plays an important role in the anticoagulation pathway. Interestingly, the serum level of TM is also correlated with disease severity in dengue patients, suggesting that the expression of TM may contribute to hemorrhage in DHF/DSS patients [67]. Taken together, the results of studies suggest that DENV infection induces MIF production, which in turn stimulates monocytes or endothelial cells to express TM and ICAM-1. Thus, MIF may play important roles not only in recruiting inflammatory cells but also in regulating coagulation. 

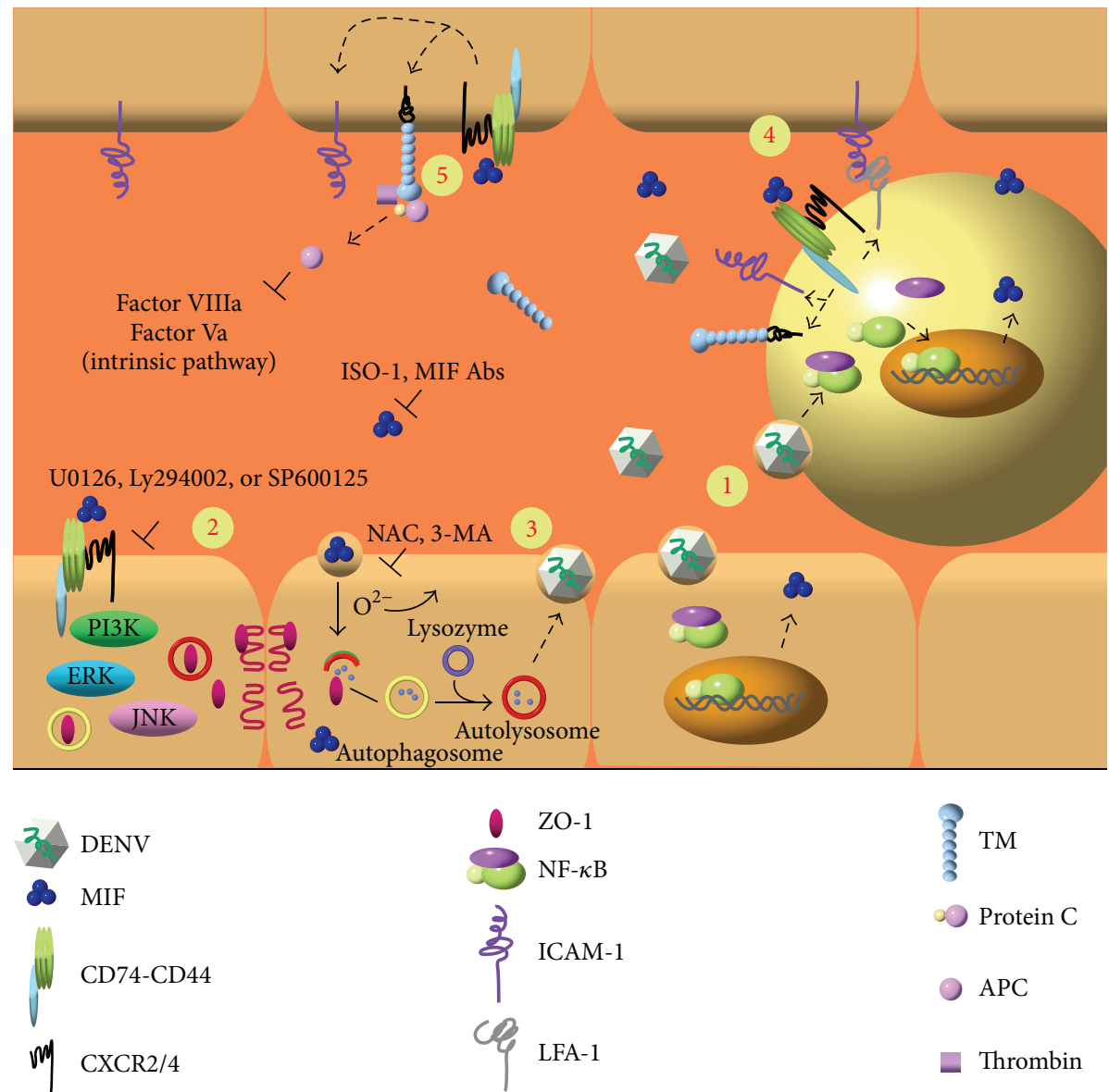

(1) DENV infection induces MIF secretion through NF- $\kappa$ B-dependent manner.

(2) MIF promotes tight junction disarray which contributes to hyperpermeability through PI3K/ERK pathway.

(3) MIF-induced autophagy could contribute to tight junction disarray and may interfere with DENV replication.

(4) MIF induces ICAM-1 and LFA-1 expression which may enhance leukocyte infiltration.

(5) MIF induces TM expression which activates protein $\mathrm{C}$ and inhibits coagulation (factor Va/VIIIa).

FIGURE 1: Schematic diagram of the pathogenic roles of MIF and possible strategies to block its effects during DENV infection. MIF expression is upregulated during DENV infection. Blocking MIF function by a MIF inhibitor (ISO-1) or MIF antibodies or its signal transduction by U0126 (Erk inhibitor), Ly294002 (PI3K inhibitor), or SP600125 (JNK inhibitor) may prevent MIF-induced permeability. In addition, MIFinduced autophagy may enhance DENV replication; therefore, blocking autophagy by NAC (ROS scavenger), 3-MA (autophagy inhibitor), or a MIF inhibitor (ISO-1) may reduce DENV replication. In addition, MIF induces the upregulation of ICAM-1 and LFA-1 expression, which may influence leukocyte recruitment. Finally, TM expression induced by MIF may activate protein C and inhibit thrombin formation. These effects of MIF can be blocked by its inhibitor or an antibody.

\section{Conclusion}

DENV infection poses a great threat to human health, with a considerable economic impact on tropical and subtropical areas of the world. Currently, there is no vaccine or specific drug to prevent or treat the disease. Indeed, the only means for preventing a DENV pandemic rely largely on targeting the vectors, which has limited effectiveness. The findings mentioned above suggest that DENV infection induces MIF production and secretion, which is NF- $\kappa \mathrm{B}$-dependent, and secreted MIF can enhance DENV replication and increase vascular leakage through autophagy. In addition, MIF can regulate adhesion molecule and coagulation molecule expression on the surface of endothelial and immune cells, which may contribute to inflammation and hemostatic abnormality during DENV infection. Therefore, prevention of the production of MIF or blocking its function by small molecules or anti-MIF antibodies may represent an additional approach to prevent the development of DHF/DSS (Figure 1).

The therapeutic antagonism of cytokines such as TNF$\alpha$ or IL-1 by biological agents has been indicated to be a successful strategy in certain autoimmune diseases. Because MIF is involved in a diversity of inflammatory diseases, it has the potential to be a therapeutic target in different 
inflammatory diseases and is not disease-specific. Moreover, compounds binding to the catalytic site of MIF that could be active anti-MIF drug candidates have been reported [68]. If the potential of such compounds is proven in clinical study, these compounds could be manufactured at relatively low cost compared to biological agents, which may benefit not only patients with DENV infection but also those with other inflammatory diseases.

\section{Conflict of Interests}

The authors declare that there is no conflict of interests regarding the publication of this paper.

\section{Acknowledgments}

This study was supported by grants from the National Science Council in Taiwan (NSC102-2320-B-006-025-MY3 and NSC101-2321-B-039-009-MY3) and by the Center of Infectious Disease and Signaling Research, National Cheng Kung University, Tainan, Taiwan.

\section{References}

[1] J. R. David, "Delayed hypersensitivity in vitro: its mediation by cell-free substances formed by lymphoid cell-antigen interaction," Proceedings of the National Academy of Sciences of the United States of America, vol. 56, no. 1, pp. 72-77, 1966.

[2] B. R. Bloom and B. Bennett, "Mechanism of a reaction in vitro associated with delayed-type hypersensitivity," Science, vol. 153, no. 3731 , pp. 80-82, 1966.

[3] Y. Asare, M. Schmitt, and J. Bernhagen, "The vascular biology of macrophage migration inhibitory factor (MIF). Expression and effects in inflammation, atherogenesis and angiogenesis," Thrombosis and Haemostasis, vol. 109, no. 3, pp. 391-398, 2013.

[4] H.-W. Sun, J. Bernhagen, R. Bucala, and E. Lolis, "Crystal structure at 2.6-A resolution of human macrophage migration inhibitory factor," Proceedings of the National Academy of Sciences of the United States of America, vol. 93, no. 11, pp. 51915196, 1996.

[5] T. Calandra, J. Bernhagen, R. A. Mitchell, and R. Bucala, "The macrophage is an important and previously unrecognized source of macrophage migration inhibitory factor," The Journal of Experimental Medicine, vol. 179, no. 6, pp. 1895-1902, 1994.

[6] T. Kumagi, F. Akbar, N. Horiike, and M. Onji, "Increased serum levels of macrophage migration inhibitory factor in alcoholic liver diseases and their expression in liver tissues," Clinical Biochemistry, vol. 34, no. 3, pp. 189-193, 2001.

[7] R. S. Gomez, T. L. Diepgen, C. Neumann, and C. Sorg, "Detection of migration inhibitory factor (MIF) by a monoclonal antibody in the microvasculature of inflamed skin," Archives of Dermatological Research, vol. 282, no. 6, pp. 374-378, 1990.

[8] T. Calandra and T. Roger, "Macrophage migration inhibitory factor: a regulator of innate immunity," Nature Reviews Immunology, vol. 3, no. 10, pp. 791-800, 2003.

[9] T. Calandra, J. Bernhagen, C. N. Metz et al., "MIF as a glucocorticoid-induced modulator of cytokine production," Nature, vol. 377, no. 6544, pp. 68-71, 1995.

[10] T. Calandra and R. Bucala, "Macrophage migration inhibitory factor: a counter-regulator of glucocorticoid action and critical mediator of septic shock," Journal of Inflammation, vol. 47, no. 1-2, pp. 39-51, 1995.

[11] S. C. Donnelly and R. Bucala, "Macrophage migration inhibitory factor: a regulator of glucocorticoid activity with a critical role in inflammatory disease," Molecular Medicine Today, vol. 3, no. 11, pp. 502-507, 1997.

[12] T. Strüßmann, S. Tillmann, T. Wirtz, R. Bucala, P. von Hundelshausen, and J. Bernhagen, "Platelets are a previously unrecognised source of MIF," Thrombosis and Haemostasis, vol. 110, no. 5, pp. 1004-1013, 2013.

[13] L. Leng, C. N. Metz, Y. Fang et al., "MIF signal transduction initiated by binding to CD74," The Journal of Experimental Medicine, vol. 197, no. 11, pp. 1467-1476, 2003.

[14] X. Shi, L. Leng, T. Wang et al., "CD44 is the signaling component of the macrophage migration inhibitory factor-CD74 receptor complex," Immunity, vol. 25, no. 4, pp. 595-606, 2006.

[15] M. Tarnowski, K. Grymula, R. Liu et al., "Macrophage migration inhibitory factor is secreted by rhabdomyosarcoma cells, modulates tumor metastasis by binding to CXCR4 and CXCR7 receptors and inhibits recruitment of cancer-associated fibroblasts," Molecular Cancer Research, vol. 8, no. 10, pp. 1328-1343, 2010.

[16] V. Schwartz, H. Lue, S. Kraemer et al., "A functional heteromeric MIF receptor formed by CD74 and CXCR4," FEBS Letters, vol. 583, no. 17, pp. 2749-2757, 2009.

[17] H. Lue, A. Kapurniotu, G. Fingerle-Rowson et al., "Rapid and transient activation of the ERK MAPK signalling pathway by macrophage migration inhibitory factor (MIF) and dependence on JAB1/CSN5 and Src kinase activity," Cellular Signalling, vol. 18, no. 5, pp. 688-703, 2006.

[18] H. Lue, M. Thiele, J. Franz et al., "Macrophage migration inhibitory factor (MIF) promotes cell survival by activation of the Akt pathway and role for CSN5/JAB1 in the control of autocrine MIF activity," Oncogene, vol. 26, no. 35, pp. 50465059, 2007.

[19] J. Bernhagen, R. Krohn, H. Lue et al., "MIF is a noncognate ligand of CXC chemokine receptors in inflammatory and atherogenic cell recruitment," Nature Medicine, vol. 13, no. 5, pp. 587596, 2007.

[20] M. Chatterjee, O. Borst, B. Walker et al., "Macrophage migration inhibitory factor limits activation-induced apoptosis of platelets via CXCR7-dependent akt signaling," Circulation Research, vol. 115, no. 11, pp. 939-949, 2014.

[21] R. Kleemann, A. Hausser, G. Geiger et al., "Intracellular action of the cytokine MIF to modulate AP-1 activity and the cell cycle through Jab1," Nature, vol. 408, no. 6809, pp. 211-216, 2000.

[22] E. F. Morand and M. Leech, "Macrophage migration inhibitory factor in rheumatoid arthritis," Frontiers in Bioscience, vol. 10, no. 1, pp. 12-22, 2005.

[23] J. Bernhagen, T. Calandra, R. A. Mitchell et al., "MIF is a pituitary-derived cytokine that potentiates lethal endotoxaemia," Nature, vol. 365, no. 6448, pp. 756-759, 1993.

[24] M. Bozza, A. R. Satoskar, G. Lin et al., "Targeted disruption of migration inhibitory factor gene reveals its critical role in sepsis," The Journal of Experimental Medicine, vol. 189, no. 2, pp. 341-346, 1999.

[25] F. A. Bozza, R. N. Gomes, A. M. Japiassú et al., "Macrophage migration inhibitory factor levels correlate with fatal outcome in sepsis," Shock, vol. 22, no. 4, pp. 309-313, 2004.

[26] Y. Al-Abed, D. Dabideen, B. Aljabari et al., "ISO-1 binding to the tautomerase active site of MIF inhibits its pro-inflammatory 
activity and increases survival in severe sepsis," The Journal of Biological Chemistry, vol. 280, no. 44, pp. 36541-36544, 2005.

[27] T. Calandra, B. Echtenacher, D. Le Roy et al., "Protection from septic shock by neutralization of macrophage migration inhibitory factor," Nature Medicine, vol. 6, no. 2, pp. 164-170, 2000.

[28] M. G. Cavalcanti, J. S. Mesquita, K. Madi et al., "Mif participates in Toxoplasma gondii-induced pathology following oral infection," PLoS ONE, vol. 6, no. 9, Article ID e25259, 2011.

[29] C. Herder, T. Illig, J. Baumert et al., "Macrophage migration inhibitory factor (MIF) and risk for coronary heart disease: results from the MONICA/KORA Augsburg case-cohort study, 1984-2002," Atherosclerosis, vol. 200, no. 2, pp. 380-388, 2008.

[30] I. I. Müller, K. A. L. Müller, H. Schönleber et al., "Macrophage migration inhibitory factor is enhanced in acute coronary syndromes and is associated with the inflammatory response," PloS ONE, vol. 7, no. 6, Article ID e38376, 2012.

[31] A. Zernecke, J. Bernhagen, and C. Weber, "Macrophage migration inhibitory factor in cardiovascular disease," Circulation, vol. 117, no. 12, pp. 1594-1602, 2008.

[32] K. D. Simpson, D. J. Templeton, and J. V. Cross, "Macrophage migration inhibitory factor promotes tumor growth and metastasis by inducing myeloid-derived suppressor cells in the tumor microenvironment," The Journal of Immunology, vol. 189, no. 12, pp. 5533-5540, 2012.

[33] E. F. Morand, M. Leech, and J. Bernhagen, "MIF: a new cytokine link between rheumatoid arthritis and atherosclerosis," Nature Reviews Drug Discovery, vol. 5, no. 5, pp. 399-411, 2006.

[34] X. Xu, B. D. Pacheco, L. Leng, R. Bucala, and J. Ren, "Macrophage migration inhibitory factor plays a permissive role in the maintenance of cardiac contractile function under starvation through regulation of autophagy," Cardiovascular Research, vol. 99, no. 3, pp. 412-421, 2013.

[35] X. Q. Hou, Y. W. Gao, S. T. Yang, C. Y. Wang, Z. Y. Ma, and X. Z. Xia, "Role of macrophage migration inhibitory factor in influenza H5N1 virus pneumonia," Acta Virologica, vol. 53, no. 4, pp. 225-231, 2009.

[36] J. Delaloye, I. J. A. de Bruin, K. E. A. Darling et al., "Increased macrophage migration inhibitory factor (MIF) plasma levels in acute HIV-1 infection," Cytokine, vol. 60, no. 2, pp. 338-340, 2012.

[37] N. Wauquier, P. Becquart, C. Padilla, S. Baize, and E. M. Leroy, "Human fatal zaire ebola virus infection is associated with an aberrant innate immunity and with massive lymphocyte apoptosis," PLoS Neglected Tropical Diseases, vol. 4, no. 10, article e837, 2010.

[38] I. Assunção-Miranda, F. A. Amaral, F. A. Bozza et al., "Contribution of macrophage migration inhibitory factor to the pathogenesis of dengue virus infection," The FASEB Journal, vol. 24, no. 1, pp. 218-228, 2010.

[39] L.-C. Chen, H.-Y. Lei, C.-C. Liu et al., "Correlation of serum levels of macrophage migration inhibitory factor with disease severity and clinical outcome in dengue patients," American Journal of Tropical Medicine and Hygiene, vol. 74, no. 1, pp. 142147, 2006.

[40] E. A. Henchal and J. R. Putnak, “The dengue viruses," Clinical Microbiology Reviews, vol. 3, no. 4, pp. 376-396, 1990.

[41] S. B. Halstead and E. J. O'Rourke, "Antibody enhanced dengue virus infection in primate leukocytes," Nature, vol. 265, pp. 739741, 1977.

[42] A. L. Rothman and F. A. Ennis, "Immunopathogenesis of dengue hemorrhagic fever," Virology, vol. 257, no. 1, pp. 1-6, 1999.
[43] H.-Y. Lei, T.-M. Yeh, H.-S. Liu, Y.-S. Lin, S.-H. Chen, and C.-C. Liu, "Immunopathogenesis of dengue virus infection," Journal of Biomedical Science, vol. 8, no. 5, pp. 377-388, 2001.

[44] V. A. Bokisch, F. H. Top Jr., P. K. Russell, F. J. Dixon, and H. J. Müller-Eberhard, "The potential pathogenic role of complement in dengue hemorrhagic shock syndrome," The New England Journal of Medicine, vol. 289, no. 19, pp. 996-1000, 1973.

[45] Y.-C. Chuang, H.-Y. Lei, H.-S. Liu, Y.-S. Lin, T.-F. Fu, and T.-M. Yeh, "Macrophage migration inhibitory factor induced by dengue virus infection increases vascular permeability," Cytokine, vol. 54, no. 2, pp. 222-231, 2011.

[46] L.-C. Chen, H.-W. Shyu, H.-Y. Lei et al., "Dengue virus infection induced NF- $\kappa$ B-dependent macrophage migration inhibitory factor production," The American Journal of Infectious Diseases, vol. 4, no. 1, pp. 22-31, 2008.

[47] T. Chareonsirisuthigul, S. Kalayanarooj, and S. Ubol, "Dengue virus (DENV) antibody-dependent enhancement of infection upregulates the production of anti-inflammatory cytokines, but suppresses anti-DENV free radical and pro-inflammatory cytokine production, in THP-1 cells," Journal of General Virology, vol. 88, no. 2, pp. 365-375, 2007.

[48] E. G. Regis, V. Barreto-de-Souza, M. G. Morgado et al., "Elevated levels of macrophage migration inhibitory factor (MIF) in the plasma of HIV-1-infected patients and in HIV-1-infected cell cultures: a relevant role on viral replication," Virology, vol. 399, no. 1, pp. 31-38, 2010.

[49] L. J. Herrero, M. Nelson, A. Srikiatkhachorn et al., "Critical role for macrophage migration inhibitory factor (MIF) in Ross River virus-induced arthritis and myositis," Proceedings of the National Academy of Sciences of the United States of America, vol. 108, no. 29, pp. 12048-12053, 2011.

[50] A. Arjona, H. G. Foellmer, T. Town et al., "Abrogation of macrophage migration inhibitory factor decreases West Nile virus lethality by limiting viral neuroinvasion," The Journal of Clinical Investigation, vol. 117, no. 10, pp. 3059-3066, 2007.

[51] Y.-R. Lee, H.-Y. Lei, M.-T. Liu et al., "Autophagic machinery activated by dengue virus enhances virus replication," Virology, vol. 374, no. 2, pp. 240-248, 2008.

[52] N. S. Heaton and G. Randall, "Dengue virus-induced autophagy regulates lipid metabolism," Cell Host and Microbe, vol. 8, no. 5, pp. 422-432, 2010.

[53] Y.-R. Lee, H.-Y. Hu, S.-H. Kuo et al., "Dengue virus infection induces autophagy: an in vivo study," Journal of Biomedical Science, vol. 20, no. 1, article 65, 2013.

[54] M. Djavaheri-Mergny, M. Amelotti, J. Mathieu et al., "NF$\kappa \mathrm{B}$ activation represses tumor necrosis factor- $\alpha$-induced autophagy," The Journal of Biological Chemistry, vol. 281, no. 41, pp. 30373-30382, 2006.

[55] J. Harris, "Autophagy and cytokines," Cytokine, vol. 56, no. 2, pp. 140-144, 2011.

[56] H.-J. Park, S. J. Lee, S.-H. Kim et al., "IL-10 inhibits the starvation induced autophagy in macrophages via class I phosphatidylinositol 3-kinase (PI3K) pathway," Molecular Immunology, vol. 48, no. 4, pp. 720-727, 2011.

[57] Y.-C. Chuang, W.-H. Su, H.-Y. Lei et al., "Macrophage migration inhibitory factor induces autophagy via reactive oxygen species generation," PLoS ONE, vol. 7, no. 5, Article ID e37613, 2012.

[58] C. A. Aveleira, C.-M. Lin, S. F. Abcouwer, A. F. Ambrósio, and D. A. Antonetti, “TNF- $\alpha$ signals through $\mathrm{PKC} \zeta / \mathrm{NF}-\kappa \mathrm{B}$ to alter the tight junction complex and increase retinal endothelial cell permeability," Diabetes, vol. 59, no. 11, pp. 2872-2882, 2010. 
[59] S. Hofmann, H. Grasberger, P. Jung et al., "The tumour necrosis factor-alpha induced vascular permeability is associated with a reduction of VE-cadherin expression," European Journal of Medical Research, vol. 7, no. 4, pp. 171-176, 2002.

[60] J. Gavard and J. S. Gutkind, "VEGF Controls endothelial-cell permeability promoting $\beta$-arrestin-dependent endocytosis VEcadherin," Nature Cell Biology, vol. 8, no. 11, pp. 1223-1234, 2006.

[61] S. Huveneers, J. Oldenburg, E. Spanjaard et al., "Vinculin associates with endothelial VE-cadherin junctions to control force-dependent remodeling," Journal of Cell Biology, vol. 196, no. 5, pp. 641-652, 2012.

[62] W. Zhu, N. R. London, C. C. Gibson et al., "Interleukin receptor activates a MYD88-ARNO-ARF6 cascade to disrupt vascular stability," Nature, vol. 492, no. 7428, pp. 252-255, 2012.

[63] H. R. Chen, Y. C. Chuang, C. H. Chao, and T. M. Yeh, "Macrophage migration inhibitory factor induces vascular leakage via autophagy," Biology Open, vol. 4, no. 2, pp. 244-252, 2015.

[64] M. A. Amin, C. S. Haas, K. Zhu et al., "Migration inhibitory factor up-regulates vascular cell adhesion molecule-1 and intercellular adhesion molecule-1 via Src, PI3 kinase, and NFkB," Blood, vol. 107, no. 6, pp. 2252-2261, 2006.

[65] L.-Y. Shyu, T.-M. Yeh, H.-H. Chang et al., "Macrophage migration inhibitory factor induces ICAM-land thrombomobulin expression in vitro," Thrombosis Research, vol. 129, no. 1, pp. 4349, 2012.

[66] T.-M. Yeh, S.-H. Liu, K.-C. Lin et al., "Dengue virus enhances thrombomodulin and ICAM-1 expression through the macrophage migration inhibitory factor induction of the MAPK and PI3K signaling pathways," PLoS ONE, vol. 8, no. 1, Article ID e55018, 2013.

[67] P. Butthep, S. Chunhakan, K. Tangnararatchakit, S. Yoksan, K. Pattanapanyasat, and A. Chuansumrit, "Elevated soluble thrombomodulin in the febrile stage related to patients at risk for dengue shock syndrome," The Pediatric Infectious Disease Journal, vol. 25, no. 10, pp. 894-897, 2006.

[68] E. F. Morand, "New therapeutic target in inflammatory disease: macrophage migration inhibitory factor," Internal Medicine Journal, vol. 35, no. 7, pp. 419-426, 2005. 


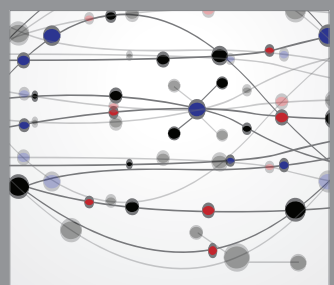

The Scientific World Journal
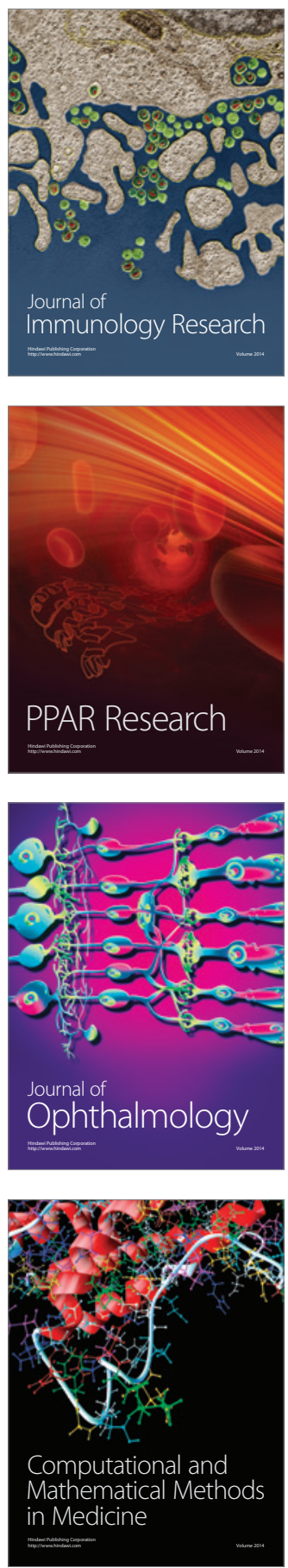

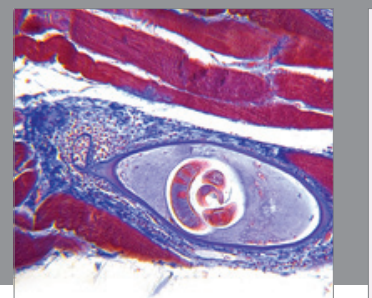

Gastroenterology

Research and Practice
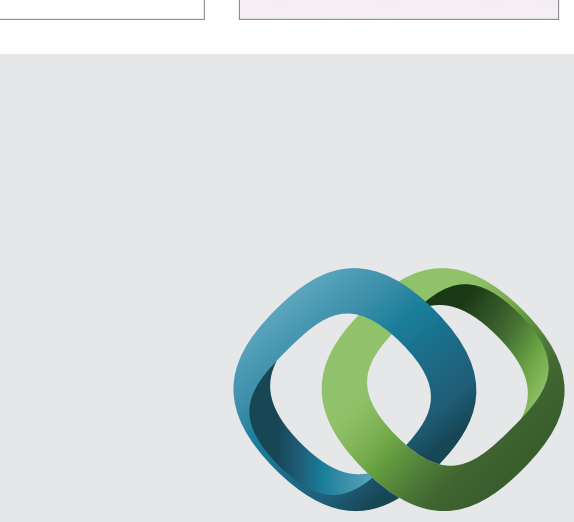

\section{Hindawi}

Submit your manuscripts at

http://www.hindawi.com
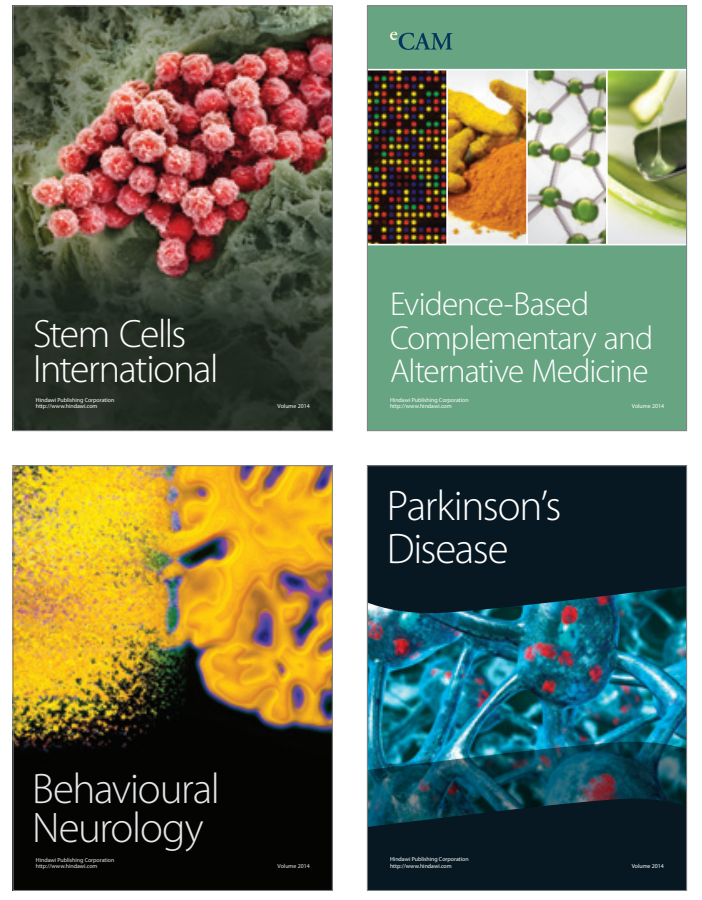
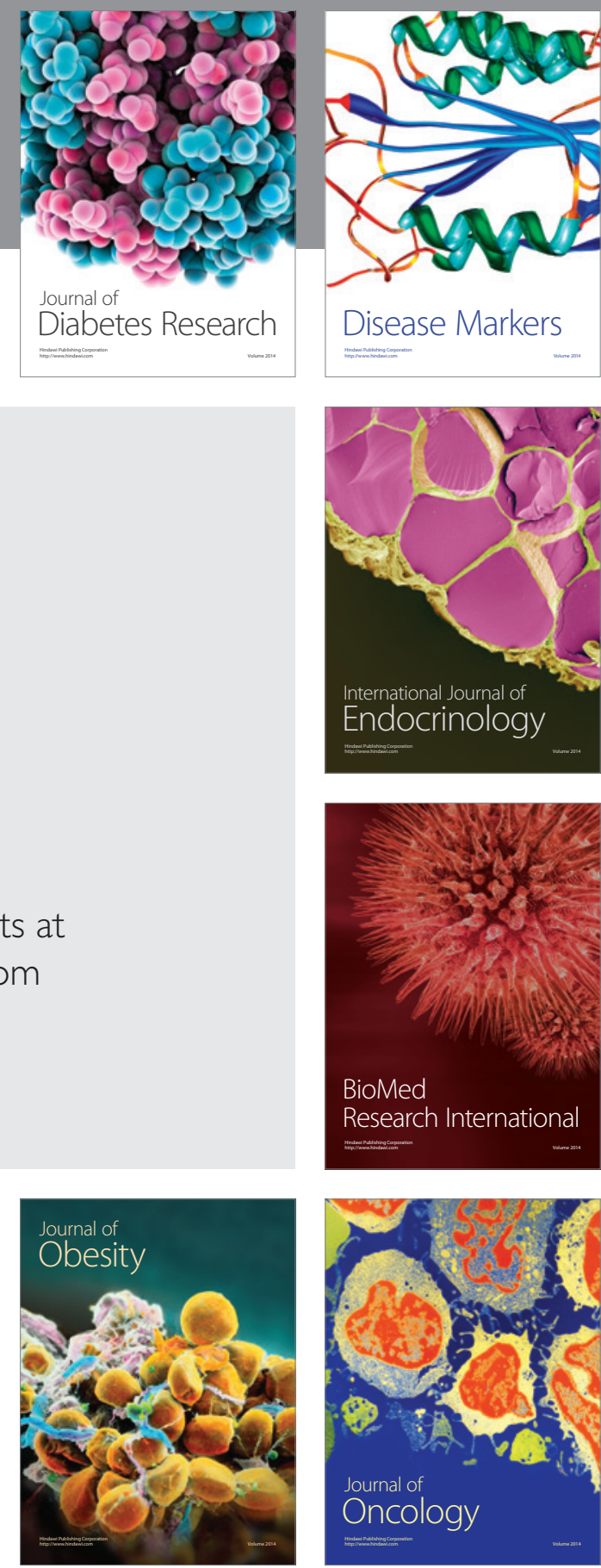

Disease Markers
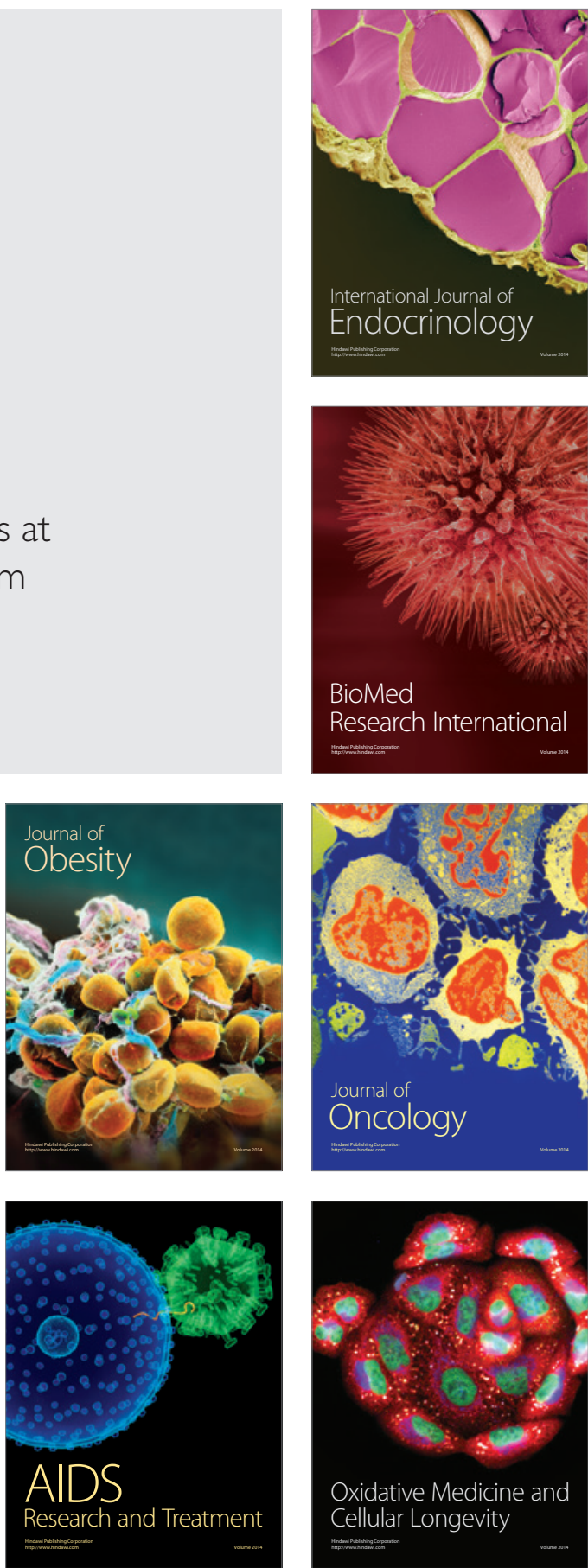